\title{
Efecto De La Humedad Relativa Y La Temperatura En La Aplicacion De Insecticidas Organofosforados Y Carbamatos E Impacto En La Colinesterasa De Agro Productores De Tomate (Solanum Lycopersicum L.) En La Localidad De "San Luis, Chimborazo, Ecuador
}

Lindao Córdova $V$. A.

Doctor en Ciencias Ambientales de la Universidad Nacional San Marcos (UNMSM) Lima Perú: Profesor ocacional Tiempo Completo de la Escuela Superior Politécnica de Chimborazo

Jave Nakayo J. L.

Doctor en Medio Ambiente y Desarrollo Sostenible, Docente de la Universidad Nacional San Marcos (UNMSM). Lima Perú

Retuerto Figueroa M. G.

Magister (c) en Medio Ambiente y Desarrollo Sostenible, Docente de la Universidad Nacional San Marcos (UNMSM) - Universidad Cesar Vallejo.

\section{Lima Perú}

Ramos Sevilla E. I.

Dr. en Ciencias Ambientales de la Universidad Nacional San Marcos (UNMSM). Profesor principal Tiempo Completo de la Escuela Superior Politécnica de Chimborazo (ESPOCH), Ecuador

Jinez Llangari P. A.

Ingeniero en Estadística Informática, Magister (c) en Estadística con Mención en control de Calidad y Productividad, Docente ocacional de la Escuela Superior Politécnica de Chimborazo, Riobamba Ecuador

Doi: 10.19044/esj.2018.v14n24p204 URL:http://dx.doi.org/10.19044/esj.2018.v14n24p204

Abstract

This research aimed to determine the effect of relative humidity and temperature in the application of organophosphorus and carbamate insecticides and impact on the colinesterasa of tomato agro producers (Solanum lycopersicum L.) in the location of" San Luis, Chimborazo, Ecuador.The technique used was spectrophotometry. The cholinesterase test was performed on the one hundred and seventy patients of the five communities exposed between one year and more than ten years, in the handling and application of organophosphorus and carbamate insecticides. The method used was kinetic-spectrophotometric $\left(405 \mathrm{~nm}\right.$ at $\left.37^{\circ} \mathrm{C}\right)$. Through 
the Kruskal Wallis test and the regression and correlation analysis it was determined the significance and the level of correlation and determination between the variables under study and their effect on cholinesterase levels. Concluding that the applications of the organophosphorus and carbamate insecticides under greenhouse with low relative humidity and high temperature, accompanied by the lack of precautions in the management of the crop, contribute to the decrease of the cholinesterase level of the agro producers.

Keywords: Relative humidity, temperature, cholinesterase, insecticides.

\section{Resumen}

La presente investigación tuvo el objetivo determinar el efecto de la humedad relativa y la temperatura en la aplicación de insecticidas organofosforados y carbamatos e impacto en la colinesterasa de agro poductores de tomate (Solanum lycopersicum L.) en la localidad de San Luis, Chimborazo - Ecuador. La técnica utilizada fue la espectrofotometría. El examen de colinesterasa se realizó a los ciento setenta pacientes de las cinco comunidades expuestos entre un año hasta más de diez años, en la manipulación y aplicación de los insecticidas organofosforados y carbamatos. El método utilizado fue el cinético-espectrofotométrico $\left(405 \mathrm{~nm}\right.$ a $\left.37^{\circ} \mathrm{C}\right)$. Mediante la prueba de Kruskal Wallis y el análisis de regresión y correlación se determinó la significancia y el grado de correlación y determinación existente entre las variables en estudio y su efecto en los niveles de colinesterasa. Concluyendo que las aplicaciones de los insecticidas organofosforados y carbamatos bajo invernadero con baja humedad relativa y temperatura alta, acompañado con la falta de precauciones en el manejo del cultivo coadyuvan la disminución del nivel de colinesterasa de los agro productores.

Palabras clave: Humedad relativa, temperatura, colinesterasa, insecticidas.

\section{Introduccion}

El desarrollo económico y social de las poblaciones conlleva a la necesidad de mejorar los sistemas de abastecimiento de alimentos, por lo que demanda un incremento en la producción, requiriendo el uso de agroquímicos para este propósito (FAO, 2106).

El control de insectos plaga en la agricultura ha dependido, en gran medida, del uso de productos químicos sintéticos que aniquilan rápidamente al insecto. Aunque este método contribuye a mantener las poblaciones plaga a niveles tolerables, su uso indiscriminado ha ocasionado varios problemas, entre ellos: la contaminación del suelo y mantos freáticos, efectos tóxicos 
en animales y el hombre, genotipos resistentes y muerte al mismo tiempo de los enemigos naturales de las mismas plagas y de otros organismos que ante la ausencia de sus reguladores se convierten en plagas secundarias (Abata, 2006).

Los productos con toxicidad aguda elevada son los responsables de un elevado número de casos de intoxicación inmediata, sobre todo en los países en desarrollo, mientras que los productos con efectos tóxicos crónicos pueden provocar cáncer o trastornos del desarrollo en niños en fase de crecimiento (FAO, 2106).

En particular, los pequeños agricultores en los países en desarrollo no suelen tener, ni utilizar, el equipo de protección necesario y acostumbran a usar pulverizadores de mochila que conllevan un alto riesgo de exposición. Limitar la utilización de estos productos muy peligrosos suele ser difícil, por lo que acaban siendo empleados de forma generalizada por personas no cualificadas. Entre las posibles consecuencias, numerosos casos de intoxicación, contaminación de alimentos y daños medioambientales. (FAO, 2106).El manejo inadecuado de plaguicidas, especialmente de organofosforados y carbamatos ocasionan serios problemas a la salud de la población y daños sobre los ecosistemas. (Karam, 2004).La humedad relativa varía inversamente con la temperatura, para un contenido absoluto de vapor de agua constante en el aire. Conforme la temperatura decrece en el invernadero la humedad relativa aumenta y puede alcanzar valores próximos a la saturación. (Huertas, 2008).La disminución en el nivel de colinesterasa en los agricultores que fumigan con humedades relativas bajas se debe a que cuando mayor sea la capacidad del aire para absorber el vapor, mejor funciona el sistema de evapotranspiración, mecanismo de regulación de la temperatura del cuerpo, aunque si es excesivamente baja, se secan las mucosas (nariz, boca) y es más propenso a la entrada de micro organismos patógenos y de agentes extraños. El grado de humedad más adecuado para la comodidad del ser humano está comprendido entre 40-70\%. (Angaramo, 2008).

En la localidad de San Luis, Chimborazo, Ecuador, la población desde hace una década aproximadamente, se ha dedicado al cultivo intensivo de tomate bajo condiciones de invernadero. Este cultivo, se ha visto afectado por el ataque de varias plagas, lo cual ha obligado la aplicación exagerada de plaguicidas especialmente del grupo de los organofosforados y carbamatos y que al no considerar factores como la humedad relativa y temperatura en el interior del invernadero al momento de la aplicación de los insecticidas pueden afectar seriamente la salud de los agricultores, población aledaña y contaminación del ambiente. Por lo que resulta importante determinar si la disminución de la humedad relativa y el incremento de la temperatura afectan los niveles de colinesterasa. Las hipótesis planteadas para esta investigación fueron: $\mathbf{H}_{\mathbf{o}}=\mathrm{El}$ nivel de colinesterasa en agro productores de tomate bajo 
invernadero en la localidad de San Luis no se ve afectado por la disminución de la humedad relativa y el incremento de la temperatura. $\mathbf{H}_{\mathbf{1}}=\mathrm{El}$ nivel de colinesterasa en agro productores de tomate bajo invernadero en la localidad de San Luis se ve afectado por la disminución de la humedad relativa y el incremento de la temperatura.

\section{Materiales Y Métodos}

El área en estudio está ubicada en los invernaderos de las cinco comunidades: Candelaria ( $\left.1^{\circ} 42^{\prime} 51.45^{\prime \prime} \mathrm{S}, 78^{\circ} 38^{\prime} 59.04^{\prime \prime} \mathrm{O}\right)$, San Antonio (1 ${ }^{\circ}$ 43'37.83" S, 78 38'56.23" O), Guaslán Grande (1 ${ }^{\circ} 43^{\prime} 37.00^{\prime}$ ' S, 78 39'15.03” O), Corazón de Jesús ( $1^{\circ} 44^{\prime}$ '08.36" S, 78 38'41.80” O), y Tiazo (1'43'03.67' S, 78 38'29.04" O), pertenecientes a la localidad de San Luis, Chimborazo, Ecuador.

La lectura del porcentaje de humedad relativa y temperatura se lo realizó con la ayuda de un higrotermómetro ubicado en el interior de cada uno de los invernaderos, los datos se registraron tomando en cuenta también la hora cuando el agricultor procedía a la aplicación de los plaguicidas. La obtención de las muestras de $5 \mathrm{ml}$ de sangre de los 170 pacientes distribuidos en las cinco comunidades seleccionadas (ver Tabla 1), se obtuvo mediante extracción por punción venosa del antebrazo en una sola sesión antes de la exposición. Todas las muestras fueron correctamente rotuladas, en completa asepsia y en tubos sin anticoagulante, con gel activador del coagulo por el método de vacutainer. (Zambonino, 2015).

Tabla 1. Características de los agros productores de la localidad de San Luis

\begin{tabular}{|c|c|c|c|}
\hline EDAD/AÑOS & $\begin{array}{c}\text { HOMBRES } \\
\text { FRECUENCIA }\end{array}$ & $\begin{array}{c}\text { MUJERES } \\
\text { FRECUENCIA }\end{array}$ & $\begin{array}{c}\text { TIEMPO DE TRABAJO EN EL } \\
\text { CAMPO Y APLICANDO } \\
\text { PLAGUICIDAS }\end{array}$ \\
\hline Entre 15 a 25 & 7 & 11 & Entre 1 a más de 10 años \\
\hline Entre 26 a 45 & 67 & 47 & Entre 1 a más de 10 años \\
\hline Entre 46 a 60 & 21 & 10 & Entre 1 a más de 10 años \\
\hline Mayores de 60 años & 5 & 2 & Entre 1 a más de 10 años \\
\hline TOTAL & $\mathbf{1 0 0}$ & $\mathbf{7 0}$ & Entre 1 a más de 10 años \\
\hline
\end{tabular}

Fuente: Lindao V. 2015

Tabla 2. Valores de referencia de la colinesterasa del grupo control

\begin{tabular}{|l|c|}
\hline \multicolumn{1}{|c|}{ Género, edad y condición } & U/L \\
\hline Niños, hombres y mujeres mayores de 40 años & $4829-11045$ \\
\hline Mujeres de 16 a 39 años, no embarazadas & $3997-9329$ \\
\hline
\end{tabular}

Fuente: Lindao V. 2016

Las muestras de sangre para determinar los valores de referencia de colinesterasa del grupo control, fueron tomadas en habitantes de las comunidades en estudio que no se dedican a las labores agrícolas (ver Tabla 2). Una vez extraídas las muestras, para su trasportarlas al laboratorio fueron colocadas en un envase tecnopor, el mismo que mantiene una temperatura de 
$4^{\circ} \mathrm{C}$ permitiendo mantener un máximo de 2 horas después de la extracción sin dañar las enzimas (Zambonino, 2015).

En el laboratorio para la obtención del suero, las muestras fueron centrifugadas a $3000 \mathrm{rpm}$ durante 10 minutos; las fases se separaron con una micro pipeta de 1000 ul el suero obtenido fue rotulado y sometido a refrigeración a temperaturas que fluctúan entre 2 a $5^{\circ} \mathrm{C}$ para luego ser analizadas (Zambonino, 2015).

El examen de colinesterasa se realizó a los pacientes expuestos a los insecticidas organofosforados y carbamatos. La técnica utilizada es la espectrofotometría que es el análisis óptico más usado por el método enzimático y cinético. La velocidad de la coloración amarilla es proporcional a la actividad enzimática y se mide a $405 \mathrm{~nm}$ a una temperatura de $37^{\circ} \mathrm{C}$. (Zambonino, 2015).

El tipo de investigación del presente trabajo fue cuasi experimental ya que, sin manipular ninguna variable, se analizó el efecto de la temperatura y la humedad relativa en la aplicación de los insecticidas organofosforados y carbamatos y su impacto en el nivel de colinesterasa en agro productores de tomate en la localidad San Luis, Chimborazo Ecuador.

Para Identificar los factores de riesgos químicos por intoxicación de carbamatos y organofosforados, debido a la heterogeneidad de la población en estudio, existentes en los agro productores de tomate, se utilizó un muestreo estratificado, tomando en cuenta las siguientes variables: humedad relativa y temperatura antes de aplicar los plaguicidas, se determinó el tamaño de la muestra aplicando la fórmula de una población finita.

La unidad de análisis fue el nivel de colinesterasa en agro productores de tomate y su relación con la humedad relativa y temperatura en el invernadero antes de la aplicación de los plaguicidas en la localidad de San Luis, Chimborazo, Ecuador.

La población en estudio fue los agros productores que cultivan tomate bajo invernadero de las cinco comunidades seleccionadas de la localidad de San Luis, Chimborazo, Ecuador.

Para determinar el tamaño de la muestra se utilizó la fórmula de la población finita:

$$
\mathrm{n}=\frac{\mathrm{N} \times \mathrm{Z}^{2} \times \mathrm{p} \times \mathrm{q}}{\mathrm{e}^{2} \times(\mathrm{N}-1)+\mathrm{Z}^{2} \times \mathrm{p} \times \mathrm{q}}
$$

De una población de 1065 agro productores que se dedican a cultivar tomate en las comunidades de la localidad de San Luis, con un margen de seguridad del $95 \%$ y una precisión del 3\% se obtuvo un tamaño de muestra de 170 agro productores distribuidos en cinco comunidades que son: Candelaria 24, San Antonio 34, Guaslán Grande 48, Corazón de Jesús 40 y Tiazo 24. 


\section{Resultados Y Discusion}

Tabla 3. Análisis factorial, prueba de Kaiser-Meyer-Olkin (KMO) y Bartlett

\begin{tabular}{|l|l|r|}
\hline Medida Kaiser-Meyer-Olkin de adecuación de muestreo & 0,778 \\
\hline Prueba de esfericidad de Bartlett & Aprox. Chi-cuadrado & 5845,949 \\
\cline { 2 - 3 } & Gl & 861,000 \\
\cline { 2 - 3 } & Sig. & 0,000 \\
\hline
\end{tabular}

Fuente: Lindao V. 2016

En la prueba de Kaiser-Meyer-Oklin (KMO), se obtuvo un estadístico de 0,778 , el mismo que tiene un valor alto, lo que nos indica que existe un buen ajuste de la muestra para este tipo de análisis (ver Tabla 3).

En la prueba de esfericidad de Bartlett, se obtiene un valor de p-0,000, el mismo que por ser menor al valor de alfa al 0,01 nos proporcionan suficientes evidencias para decir que entre las variables en estudio existen correlaciones significativas (ver Tabla 3 ).

Tabla 4. Prueba de Kruskal Wallis para porcentaje de humedad relativa dentro del invernadero y nivel de colinesterasa.

\begin{tabular}{|c|c|c|c|c|}
\hline $\begin{array}{l}\text { Porcentaje de humedad } \\
\text { relativa dentro del } \\
\text { invernadero }\end{array}$ & g.l. & p - valor & $\begin{array}{l}\text { Medias nivel de } \\
\text { colinesterasa }\end{array}$ & Grupo \\
\hline 87 & 26 & $<0,0001$ & 12344,00 & A \\
\hline 85 & & & 10962,20 & A \\
\hline 84 & & & 9608,21 & A \\
\hline 78 & & & 9091,03 & A \\
\hline 77 & & & 8753,10 & A \\
\hline 76 & & & 8413,00 & A B \\
\hline 75 & & & 8311,88 & A B \\
\hline 72 & & & 8104,38 & A B \\
\hline 71 & & & 7903,33 & A B \\
\hline 70 & & & 7603,33 & A B \\
\hline 69 & & & 7112,85 & A B \\
\hline 68 & & & 7115,67 & A B C \\
\hline 67 & & & 6799,40 & A B C \\
\hline 66 & & & 6401,75 & A B C \\
\hline 65 & & & 6097,80 & $A B C D$ \\
\hline 64 & & & 5782,75 & A B C D \\
\hline 63 & & & 5109,75 & A B C D \\
\hline 40 & & & 3786,63 & A B C D \\
\hline 39 & & & 3188,21 & B C D \\
\hline 38 & & & 2788,71 & C D E \\
\hline 37 & & & 2475,43 & C D E F \\
\hline 36 & & & 2172,50 & CDEF \\
\hline 35 & & & 1932,00 & CDEF \\
\hline 31 & & & 1692,33 & D E F \\
\hline 33 & & & 1496,74 & E F \\
\hline 32 & & & 1476,81 & EF \\
\hline 29 & & & 1188,95 & $\mathrm{~F}$ \\
\hline
\end{tabular}

Fuente: Lindao, V. 2016.

$$
\begin{gathered}
\mathrm{p}<0,05 * \\
\mathrm{p}<0,01 * * \\
\mathrm{p}>0,05,>0,01 \mathrm{~ns}
\end{gathered}
$$


En la Prueba de Kruskal Wallis al 5\%, para humedad relativa dentro del invernadero y nivel de colinesterasa se observa diferencias altamente significativas con un p-valor de $<0,0001$.

En la separación de medias se obtiene diez grupos, en el grupo A se ubican los agro productores que fumigan los insecticidas con humedades relativas de $87,85,84,78,77$, \% con medias de 12344,00, 10962,20, 9608,21, 9091,03 y 8753,28 U/1, respectivamente, en el grupo F se encuentra los agro productores que fumigan los insecticidas con una humedad relativa de $29 \%$ con una media de 1188,95 U/l (ver Tabla 4).

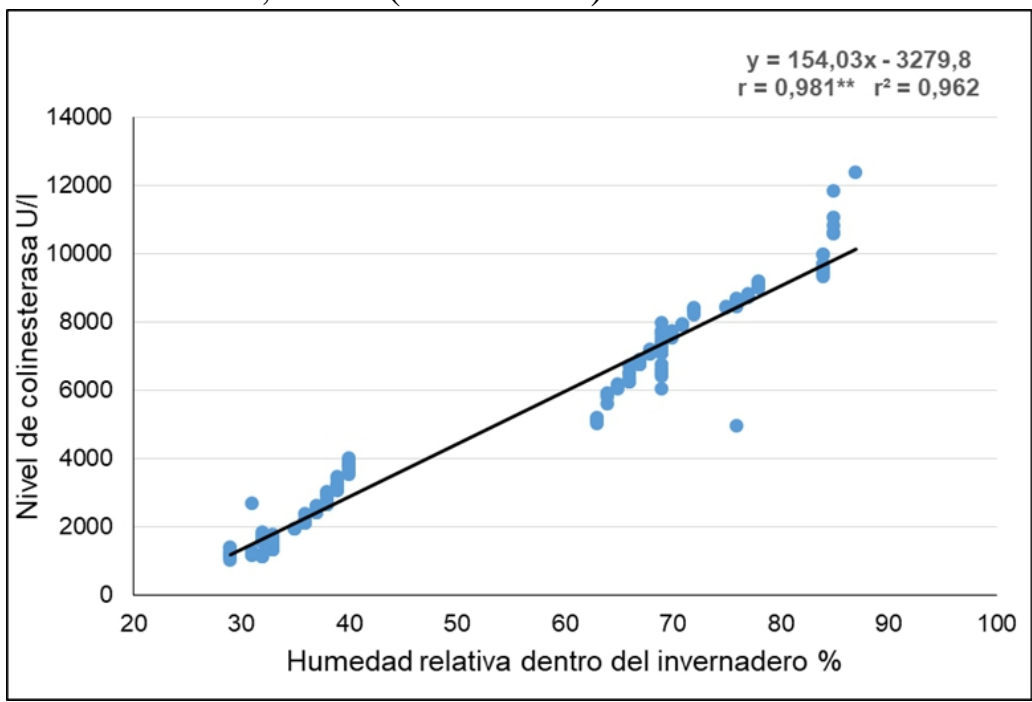

Figura 1. Análisis de regresión y correlación para humedad relativa dentro del invernadero y nivel de colinesterasa. Fuente: Lindao V. 2016.

En la figura 1, se puede apreciar que existe una correlación significativa al 0,01 entre las variables humedad relativa dentro del invernadero y nivel de colinesterasa con un valor de $r=0,981^{* *}$, la relación que presenta es directamente proporcional, como producto del incremento de las dos variables. El coeficiente de determinación $\mathrm{r}^{2}=0,962$ nos indica que el $96,2 \%$ del nivel de colinesterasa depende de la humedad relativa dentro del invernadero el restante $3,8 \%$ depende de otros factores.

La humedad relativa es directamente proporcional al nivel de colinesterasa, en cambio con la temperatura presenta una relación inversa, lo que concuerda (Huertas, 2008), quien manifiesta que la humedad relativa varía inversamente con la temperatura, para un contenido absoluto de vapor de agua constante en el aire. Conforme la temperatura decrece en el invernadero la humedad relativa aumenta y puede alcanzar valores próximos a la saturación.

La disminución en el nivel de colinesterasa en los agro productores que fumigan con humedades relativas bajas se debe a que cuando mayor sea la 
capacidad del aire para absorber el vapor, mejor funciona el sistema de evapotranspiración, mecanismo de regulación de la temperatura del cuerpo, aunque si es excesivamente baja, se secan las mucosas (nariz, boca) y es más propenso a la entrada de micro organismos patógenos y de agentes extraños. El grado de humedad más adecuado para la comodidad del ser humano está comprendido entre 40-70\% (Angaramo, 2008), estos resultados coinciden con los obtenidos en la investigación, en donde los niveles más bajos de colinesterasa se presenta cuando la humedad relativa baja del $40 \%$.

En la Prueba de Kruskal Wallis al 5\% (ver Tabla 5), para temperatura dentro del invernadero y nivel de colinesterasa se observa diferencias altamente significativas con un p-valor de $<0,0001$.

En la separación de medias se obtiene cinco grupos, en el grupo A se ubican los agricultores que fumigan los insecticidas con temperatura de 8,9 , $10,14,15,17,16,19,20$ y $21^{\circ} \mathrm{C}$ con medias de $12344,00,10962,20,9608,21$, $9091,03,8753,10,8413,00,8104,00,7976,75,5940,28$ y 5542,28 U/1, respectivamente, en el grupo $\mathrm{D}$ se encuentran los agricultores que fumigan los insecticidas con una temperatura de $39{ }^{\circ} \mathrm{C}$ con una media de $1392,99 \mathrm{U} / \mathrm{l}$ (ver Tabla 5).

Tabla 5. Prueba de Kruskal Wallis al 5\% y separación de medias para temperatura dentro del invernadero y nivel de colinesterasa

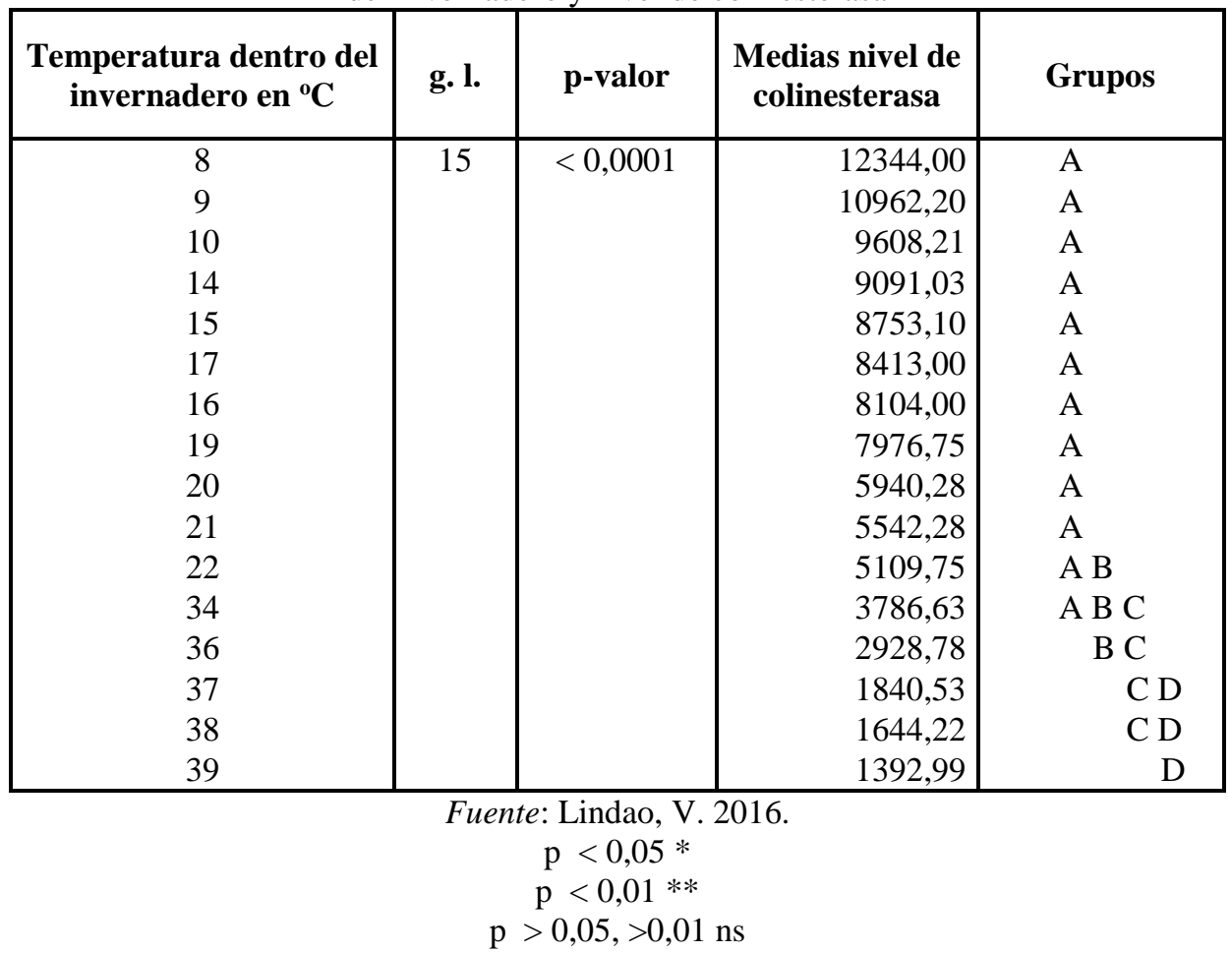


Como se puede apreciar en la (Tabla 5) la aplicación de los insecticidas con temperaturas de $39{ }^{\circ} \mathrm{C}$, influyen negativamente en el nivel de colinesterasa, en cambio cuando lo realiza la aplicación con temperaturas bajas de $8{ }^{\circ} \mathrm{C}$, el nivel de colinesterasa es alto, lo que concuerda que concuerda con (Dreisbach \& Robertson, 1988), quienes manifiestan que la absorción por la piel es mayor a temperaturas más altas y mucho mayor en presencia de dermatitis.

(Mohammad, H, \& Varela, 2008), manifiestan que los insecticidas órgano fosforados pueden ingresar al organismo por inhalación de vapores, vacíos o polvos, por absorción gastrointestinal y aún por penetración a través de la piel y de las mucosas expuestas.

Los resultados obtenidos también coinciden con (Buedo, 2013), quien señala que en el trabajo con productos químicos la temperatura ambiental es muy importante, pudiendo decir que conforme aumenta la temperatura aumentan los problemas. Cuando la temperatura ambiental en el trabajo en invernaderos o el esfuerzo físico es considerable, la absorción cutánea de los plaguicidas es más rápida. Al mismo tiempo, por la acción del calor aumenta la volatilización, emisión de vapores, etcétera, pudiendo aumentar la absorción a través de las vías respiratorias.

Los datos obtenidos coinciden con (Milla, 2018), quien manifiesta que la exposición a los plaguicidas afecta considerablemente los niveles de colinesterasa sérica en los agricultores expuestos a los plaguicidas

Lo manifestado anteriormente coinciden también con (Echeverria, 2008), quién indica que la velocidad de absorción de los plaguicidas depende de varios factores entre los que se incluyen: temperatura y humedad ambiental, la concentración del tóxico, la magnitud y localización en el cuerpo del área expuesta, la condición de la piel. La hidratación, quemaduras y ciertas enfermedades incrementan la permeabilidad y la velocidad del flujo sanguíneo.

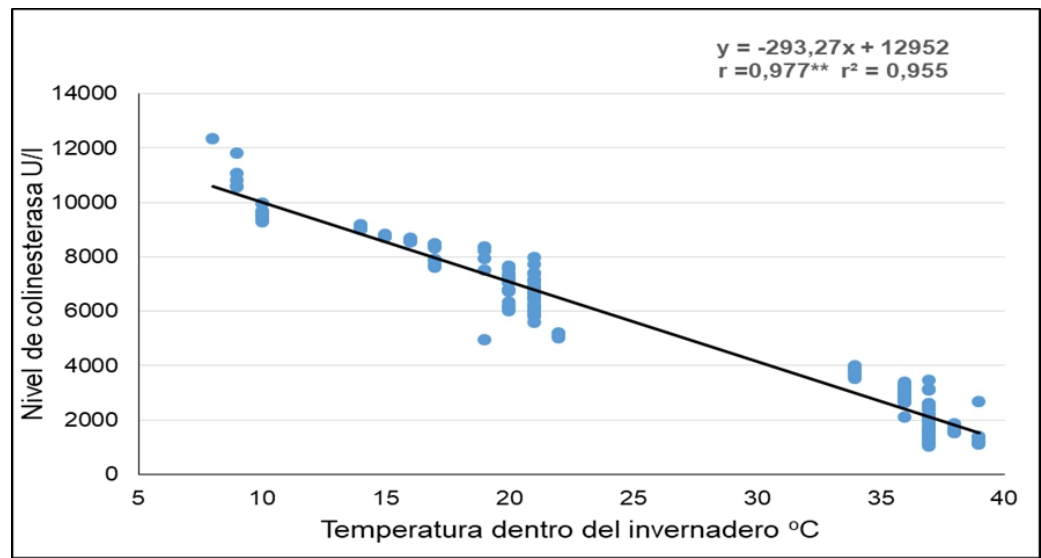

Figura 2. Análisis de regresión y correlación para temperatura dentro del invernadero y nivel de colinesterasa. Fuente: Lindao V. 2016. 
Se puede apreciar que existe una correlación significativa al 0,01 entre las variables temperatura dentro del invernadero y nivel de colinesterasa con un valor de $\mathrm{r}=0,977^{* *}$, la relación que presenta es inversamente proporcional, como producto del incremento de la variable temperatura y el decremento del nivel de colinesterasa. El coeficiente de determinación $\mathrm{r}^{2}=0,955$ nos indica que el $95,5 \%$ del nivel de colinesterasa depende de la temperatura dentro del invernadero el restante 4,5\% depende de otros factores (ver Figura 2).

La (Universidad Técnica de Cataluña, 2016), manifiesta que las vías de absorción más importantes son la cutánea (sin duda la más importante) y la respiratoria. La vía digestiva no es muy frecuente, pero cuando ocurre está asociada al incumplimiento de los protocolos de higiene personal (comer, beber o fumar en el trabajo sin lavarse previamente las manos y la cara). Los plaguicidas son compuestos que 'poseen una enorme facilidad para disolverse en las grasas, motivo por el que la piel, donde se encuentra una importante capa de tejido con elevado contenido de lípidos, constituyendo una importante puerta de entrada. Este autor indica que el aumento de temperatura favorece la absorción cutánea de los plaguicidas, lo que concuerda con Los resultados obtenidos en el análisis de regresión en donde se observa que a medida que se eleva la temperatura el nivel de la enzima baja (ver Figura 2).

Aceptamos la $\mathbf{H}_{1}$ = dado que el nivel de colinesterasa en agricultores de tomate bajo invernadero en la localidad de San Luis, se ve afectado por la disminución de la humedad relativa, la relación es directamente proporcional, a medida que la humedad relativa aumenta el nivel de colinesterasa es mayor, con un coeficiente de correlación $\mathrm{r}=0,981^{*} *$, en cambio la relación de la temperatura con los niveles de colinesterasa, es inversamente proporcional dado que cuando la temperatura aumenta el nivel de colinesterasa disminuye con un coeficiente de correlación $\mathrm{r}=-0,977^{*} *$.

\section{Conclusion}

Las aplicaciones de los insecticidas organofosforados y carbamatos bajo invernadero con baja humedad relativa $29 \%$ y temperatura alta $39^{\circ} \mathrm{C}$, acompañado con la falta de precauciones en el manejo del cultivo, coadyuvan la disminución del nivel de colinesterasa de los agro productores de 12344,00 U/1 con una humedad relativa del $87 \%$ a $1188,95 \mathrm{U} / \mathrm{l}$ con una humedad relativa del $29 \%$ y de $12344,00 \mathrm{U} / 1$ con una temperatura de $8^{\circ} \mathrm{C}$ a $1392,99 \mathrm{U} / \mathrm{l}$ con una temperatura de $39^{\circ} \mathrm{C}$.

\section{Agradecimientos}

Agradecimientos por sus valiosos aportes brindados a la Universidad Nacional Mayor San Marcos (UNMSM), Escuela Superior Politécnica de Chimborazo (ESPOCH) Facultad de Recursos Naturales y al Dr. Jorge Jave 
quien ha sido parte fundamental en el desarrollo y culminación de este trabajo de investigación como director.

\section{References:}

1. Abata, P. (2006). ). "Determinación de los niveles Séricos de Colinesterasa en los Habitantes de la Comunidad de Yacubamba, Cantón Pujilí, Provincia de Cotopaxi debido a la utilización de Plaguicidas Químicos” (Tesis de Pregrado) Universidad Técnica del Cotopaxi, Latacunga-Ecuador.

2. Angaramo, C. (2008). Escuelas técnica IPEM 56 Abraham Juarez. Recuperado de Nuestra escuela: http://www.oni.Escuelas.edu.Ar/2008/Córdoba/1324/trabajo/ humedad ambiente.html

3. Buedo, E. (05 de 2013). Daños para la salud y factores que influyen. Obtenido de Criterios de prevención del riesgo. Conducta a seguir en caso de intoxicación por plaguicidas.Dreisbach, R. H., \& Robertson, W. O. (1988). Manual de Toxicología Clínica: prevención, diagnóstico y tratamiento. El Manual Moderno.

4. Echeverria, M. (2008). Introducción a la Toxicología Ambiental. Riobamaba: Arte Guerrotipo Digital.

5. Organización de las Naciones Unidas para la Agricultura y la Alimentación - FAO. (2016). "Lucha contra la contaminación agrícola de los recursos hídricos" Recuperado a partir de http://www.fao.org/docrep/w2598s/w2598s06.htm.

6. Huertas, L. (2008). El control ambiental en invernaderos: humedad relativa. Industria hortícola, tecnología de producción, 52-54.

7. Karam, M. Á., Ramírez, G., Bustamante Montes, L. P., \& Galván, J. M. (2004). Plaguicidas y salud de la población. CIENCIA ergo sum, 11(3).

8. 9. Milla Cotos, Oscar Manuel y Palomino Horna, William Rodolfo (2002). Niveles de colinesterasa sérica en agricultores de la localidad de Carapongo (Perú) y determinación de residuos de plaguicidas inhibidores de la aceilcolinesterasa en frutas y hortalizas cultivadas. Recuperado de http://sisbib.unmsm.edu.pe/BibVirtualData/ Tesis/Salud/Milla_C_O/discus.pdf

9. Mohammad, H, \& Varela, S. (2008). Insecticidas Organofosforados: efectos sobre la Salud y el Ambiente. Recuperado de https://www.researchgate.net/publication/

28249214_Insecticidas_Organofosforados_efectos_sobre_la_Salud_y _el_Ambiente

10. Organización de las Naciones Unidas - ONU. (1986). Organización de las Naciones Unidas para la Agricultura y la Alimentación. La 
alimentación y el medio ambiente. Desarrollo Coop (Alemania Federal) 1986;1:18-20. En O. D. ALIMENTACION, La alimentación y el medio ambiente. (págs. 18-20). Alemania Federal: Desarrollo Coop (Alemania Federal).

11. Universidad Técnica de Cataluña. (2016). Prevencion integral. Recuperado de http://www.prevencionintegral.com/ca/comunidad/blog/toxicologialaboral-peligros-riesgos/2016/07/27/insecticidasorganofosforadoscarbamatos.

12. Zambonino M. (2015). "Determinación de los niveles de colinesterasa y evaluación de la presencia de efectos neurotóxicos en trabajadores expuestos a plaguicidas Organofosforados y Carbamatos de la parroquia de San Luis". 